\title{
A cost effectiveness analysis of midwife psycho-education for fearful pregnant women - a health system perspective for the antenatal period
}

\author{
J Toohill ${ }^{1,4^{*}}$ D, E Callander ${ }^{2}$, J Gamble ${ }^{1}$, DK Creedy ${ }^{1}$ and J Fenwick ${ }^{1,3}$
}

\begin{abstract}
Background: Psycho-education can reduce childbirth fear and caesarean section numbers. This study determines the cost-effectiveness of a midwife-led psycho-education intervention for women fearful of birth.

Method: One thousand four hundred ten pregnant women in south-east Queensland, Australia were screened for childbirth fear (W-DEQ A $\geq 66$ ). Women with high scores $(n=339)$ were randomised to the BELIEF Study (Birth Emotions and Looking to Improve Expectant Fear) to receive psycho-education $(n=170)$ at 24 and 34 weeks of pregnancy or to the control group $(n=169)$. Women in both groups were surveyed 6 weeks postpartum with total cost for health service use during pregnancy calculated. Logistic regression models assessed the odds ratio of having vaginal birth or caesarean section in the study groups.
\end{abstract}

Result: Of 339 women randomised, 184 (54\%) women returned data at 6 weeks postpartum (Intervention Group $n=91$; Control Group $n=93$ ). Women receiving psycho-education had a higher likelihood of vaginal birth compared to controls ( $n=60,66 \%$ vs. $n=54,58 \% ;$ OR 2.34). Mean 'treatment' cost for women receiving psycho-education was AUS\$72. Mean cost for health services excluding the cost of psycho-education, was less in the intervention group (AUS\$1193 vs. AUS\$1236), but not significant $(p=0.78)$. For every five women who received midwife counselling, one caesarean section was averted. The incremental healthcare cost to prevent one caesarean section using this intervention was AUS\$145.

Conclusion: Costs of delivering midwife psycho-education to women with childbirth fear during pregnancy are offset by improved vaginal birth rates and reduction in caesarean section numbers.

Trial registration: Australian New Zealand Controlled Trials Registry ACTRN12612000526875, 17th May 2012 (retrospectively registered one week after enrolment of first participant).

Keywords: Childbirth fear, W-DEQ A, Cost effectiveness, Caesarean section, Midwife psycho-education

\section{Background}

Worldwide $14 \%$ of women are affected by severe levels of childbirth fear [1]. While definitions of severity may vary across cultures and studies, childbirth fear at high or severe levels has a significant effect on a woman's ability to approach birth positively and achieve an

\footnotetext{
* Correspondence: j.toohill@griffith.edu.au

${ }^{1}$ School of Nursing and Midwifery \& Menzies Health Institute Queensland, Griffith University, University Drive, Meadowbrook 4131, Australia

${ }^{4}$ Office of the Chief Nursing and Midwifery Officer, Queensland Department of Health, Brisbane 4001, Australia

Full list of author information is available at the end of the article
}

optimal birth outcome [2-4]. In resource rich countries, childbirth fear is commonly associated with maternal depression and or anxiety before $[5,6]$ and following birth [7]. Fear is influenced or exacerbated by a myriad of personal, social, and health system factors including a maternity milieu characterised by increasing rates of obstetric interventions. As a consequence, women may develop an exaggerated concern that birth will result in harm to themselves or to their baby $[8,9]$.

The overall prevalence of childbirth fear in Australian women is around $25 \%$ and as high as $32 \%$ for women having their first baby [10-12]. There is evidence that 
caesarean section occurs more frequently in women who are fearful of childbirth $[4,13,14]$, and that caesarean section increases health costs to women and maternity systems $[15,16]$. Australia has higher rates of childbirth fear compared to other developed countries [1, 10, 11], and worldwide has one of the highest rates of caesarean section [17]. Several studies have reported reduced caesarean section numbers following psycho-education interventions in women with childbirth fear [18-21] but few have reported costs associated with interventions $[13,22,23]$. We have previously reported on a cost analysis of the intervention on women's quality of life and health service usage to six weeks post birth, demonstrating no additional cost to health services following provision of antenatal midwife-led psycho-education [23]. This current paper reports on the cost of the intervention in preventing unnecessary caesarean section from a health system perspective looking specifically at costs incurred during the antenatal period. This approach was taken to provide information on the relative costs of delivering the intervention compared to standard care for decision makers responsible for healthcare delivery.

\section{Aim}

To determine the cost effectiveness of midwife-led psycho-education for fearful pregnant women, relative to standard care.

\section{Methods}

Data was drawn from the BELIEF (Birth Emotions and Looking to Improve Expectant Fear) study, a randomised controlled trial of midwife telephone psycho-education for women fearful of birth. The study protocol [24] and results of the intervention have been reported previously [25].

Women $(n=1410)$ in their second trimester of pregnancy at antenatal booking clinics across three maternity hospitals in Queensland, Australia were screened for childbirth fear using the Wijma Delivery Expectancy/Experience Questionnaire (W-DEQ A) [26]. Women with high levels of fear (W-DEQ A $\geq 66$ ) were randomised to receive telephone psycho-education at 24 and 34 weeks of pregnancy from a midwife trained in the intervention $(n=170)$ or to the control group $(n=169)$. Characteristics of the intervention and control groups are shown in Table 1. Women in the control group received usual antenatal care. Both groups also received a birth decision aid booklet. Demographic, obstetric information, birth preference and psychosocial measures were collected at recruitment and 36 weeks; with birth method and health service use returned by participants six weeks following birth. For further detail please refer to the study protocol [24].
Table 1 Characteristics of total sample of women in the intervention and control groups at baseline \& 36 weeks

\begin{tabular}{|c|c|c|}
\hline Characteristic & $\begin{array}{l}\text { Intervention Group } \\
N=170\end{array}$ & $\begin{array}{l}\text { Control Group } \\
N=169\end{array}$ \\
\hline Age (Mean) & 28.5 & 28.7 \\
\hline \multicolumn{3}{|l|}{ Level of Education Attainment } \\
\hline $\begin{array}{l}\text { Did not complete } \\
\text { year } 12\end{array}$ & $37(22 \%)$ & $32(19 \%)$ \\
\hline Completed year 12 & 49 (29\%) & $48(28 \%)$ \\
\hline Diploma & $50(29 \%)$ & $47(28 \%)$ \\
\hline Bachelor degree & $23(14 \%)$ & $33(20 \%)$ \\
\hline $\begin{array}{l}\text { Postgraduate } \\
\text { qualifications }\end{array}$ & $11(6 \%)$ & $9(5 \%)$ \\
\hline BMI (Mean) & 26.2 & 27.0 \\
\hline Nulliparous & $96(56 \%)$ & $95(56 \%)$ \\
\hline Last birth Caesarean & $27(36 \%)$ & $26(35 \%)$ \\
\hline $\begin{array}{l}\text { WDEQ-A Time1 Mean (SD), } \\
\text { range }\end{array}$ & $\begin{array}{l}80.0(\text { SD 12.4) } \\
66-127\end{array}$ & $\begin{array}{l}76.3(S D \text { 10.6) } \\
66-128\end{array}$ \\
\hline \multicolumn{3}{|l|}{36 weeks } \\
\hline $\begin{array}{l}\text { WDEQ-A Time2 } 2^{\mathrm{a}} \text { Mean (SD), } \\
\text { range }\end{array}$ & $\begin{array}{l}\text { 61.0 (SD 19.7) } \\
12-117\end{array}$ & $\begin{array}{l}66.5(\text { SD 18.2) } \\
22-121\end{array}$ \\
\hline
\end{tabular}

The time frame for this economic evaluation was the duration of pregnancy, with analysis conducted from a health system perspective. The number of caesarean sections prevented (either planned or unplanned) was chosen as the focal outcome of the cost-effectiveness analysis because this was the main clinical outcome produced by a reduction in fear of birth. Increasing caesarean section rates are a large driver of health system costs, being more resource intensive than vaginal births [27]. Women were surveyed at 6 weeks postpartum about their health service use during pregnancy. All reported health service use during the antenatal period up to the time of birth were included. Unit costs were combined with women's reported frequency of health service use during pregnancy. The unit cost assigned to each type of health service use is outlined in Table 2 .

Logistic regression models were used to assess the odds ratio of having a vaginal birth and having a caesarean section for women in the intervention and control groups. The models were adjusted for women's age and education attainment, BMI, baseline W-DEQ A score and hospital site.

The number of women needed to be treated (NNT) to prevent one caesarean section was calculated based upon the population expected event risk (PEER) for caesarean section, which was assumed to be equivalent to the Control Event Risk (CER) of women who underwent usual care, and the adjusted-odds ratio of having a caesarean section for the intervention group compared to the control group. The formula used to calculate NNT was: NNT $=(1-$ $(\operatorname{PEER} *(1-\mathrm{OR})))$ / ((1-PEER)*(PEER)*(1-OR)) [28, 29]. 
Table 2 Costs associated with health service use

\begin{tabular}{|c|c|c|}
\hline Health service use & $\begin{array}{l}\text { Unit } \\
\text { Cost }\end{array}$ & Source \\
\hline Intervention costs & $\$ 40.17$ & $\begin{array}{l}\text { Based upon Queensland Health } \\
\text { Midwife wages }\end{array}$ \\
\hline GP Visits & $\$ 36.30$ & $\begin{array}{l}\text { Medicare Benefits Schedule, } \\
\text { Item } 23\end{array}$ \\
\hline Obstetrician visits & $\$ 75.50$ & $\begin{array}{l}\text { Medicare Benefits Schedule, } \\
\text { Item } 116\end{array}$ \\
\hline Midwife visits & $\$ 40.17$ & $\begin{array}{l}\text { Based upon Queensland Health } \\
\text { Midwife wages }\end{array}$ \\
\hline Nurse visits & $\$ 40.17$ & $\begin{array}{l}\text { Based upon Queensland Health } \\
\text { Midwife wages }\end{array}$ \\
\hline Ultrasounds & $\$ 78.52$ & $\begin{array}{l}\text { Medicare Benefits Schedule, average } \\
\text { of items related to ultrasounds }\end{array}$ \\
\hline $\begin{array}{l}\text { Hospital Admissions } \\
\text { (Antenatal) }\end{array}$ & $\$ 1547.04$ & $\begin{array}{l}\text { 2013-14 National Efficient Price } \\
\text { rates produced by the Independent } \\
\text { Hospital Pricing Authority, based on } \\
\text { AR-DRG O66A, O66B }\end{array}$ \\
\hline $\begin{array}{l}\text { Emergency Department } \\
\text { visits }\end{array}$ & $\$ 570.70$ & $\begin{array}{l}\text { 2013-14 National Efficient Price } \\
\text { rates produced by the Independent } \\
\text { Hospital Pricing Authority, Triage } \\
\text { Category } 3 \text { (Obstetric/Gynecology } \\
\text { illness) }\end{array}$ \\
\hline
\end{tabular}

The incremental cost-effectiveness ratio (ICER) was estimated from the difference between the total health care costs (cost of intervention plus other health care use) of the intervention and control groups multiplied by the number of women that needed to be treated (NNT) to prevent one caesarean section.

\section{Results}

One hundred and eighty- four women returned data at six weeks following birth (Intervention $n=91$; Control $n=93)$. The characteristics of this final sample included in this study are presented in Table 3.

\section{Relationship between W-DEQ a score at 36 weeks and birth type}

Across all women the W-DEQ A score at 36 weeks gestation was generally associated with a woman's type of birth, shown in Table 4. After controlling for age, level of education attainment and hospital site, a lower WDEQ A score compared to higher scores at 36 weeks gestation was shown to be significantly related to the likelihood of having a vaginal birth as opposed to caesarean section $(-0.02, p=0.027)$.

\section{Birth type between intervention and control groups}

A larger proportion of women from the intervention group had a vaginal birth (66\%) than women in the control group (58\%) (Table 5). After adjusting for age, level of education attainment, BMI, baseline W-DEQ A score and hospital site, women in the intervention group had 2.34
Table 3 Characteristics of final sample of women with nonmissing health service use data in the intervention and control groups at baseline \& 36 weeks

\begin{tabular}{|c|c|c|}
\hline Characteristic & $\begin{array}{l}\text { Intervention Group } \\
N=91\end{array}$ & $\begin{array}{l}\text { Control Group } \\
N=93\end{array}$ \\
\hline Age (Mean) & 29.2 & 29.5 \\
\hline \multicolumn{3}{|l|}{ Level of Education Attainment } \\
\hline $\begin{array}{l}\text { Did not complete } \\
\text { year } 112\end{array}$ & $14(18 \%)$ & $9(9 \%)$ \\
\hline Complete year 12 & $23(25 \%)$ & $23(25 \%)$ \\
\hline Diploma & $25(27 \%)$ & $30(32 \%)$ \\
\hline Bachelor degree & $18(20 \%)$ & $26(28 \%)$ \\
\hline Postgraduate qualifications & $8(9 \%)$ & $5(5 \%)$ \\
\hline BMI (Mean) & 26.9 & 26.1 \\
\hline Nulliparous & $51(56 \%)$ & $53(57 \%)$ \\
\hline Last birth Caesarean & $14(18.6 \%)$ & $13(14 \%)$ \\
\hline $\begin{array}{l}\text { WDEQ-A Time1 Mean (SD), } \\
\text { range }\end{array}$ & $\begin{array}{l}82.2(\text { SD13.5) } \\
66-127\end{array}$ & $\begin{array}{l}75.3(\text { SD9.2) } \\
66-108\end{array}$ \\
\hline \multicolumn{3}{|l|}{36 weeks } \\
\hline $\begin{array}{l}\text { WDEQ-A Time2 } 2^{\mathrm{a}} \text { Mean (SD), } \\
\text { range }\end{array}$ & $\begin{array}{l}61.4(\text { SD20.6) } \\
12-117\end{array}$ & $\begin{array}{l}66.1(\mathrm{SD} 18.5) \\
22-121\end{array}$ \\
\hline $\begin{array}{l}\text { Smoked throughout } \\
\text { pregnancy }\end{array}$ & $5(5.5)$ & $2(2.2)$ \\
\hline
\end{tabular}

times the odds of having a vaginal birth than women in the control group (95\% CI: 1.16-4.73, $p=0.014$ ). Women in the intervention group had 0.41 times the odds of having a caesarean section compared to women in the control group (95\% CI: $0.20-0.85, p=0.017$ ).

Cost comparison between intervention and control groups The cost estimates are summarized in Table 6, which presents both the intervention cost and health service use cost during pregnancy. The mean 'treatment' cost for women receiving the intervention was AUS\$72. The mean cost for health service use, excluding the cost of

Table 4 Relationship between birth type and WDEQ score at 36 weeks gestation, all women

\begin{tabular}{llll}
\hline Birth type & N & $\begin{array}{l}\text { Mean WDEQ } \\
\text { score }\end{array}$ & SD \\
\hline Vaginal birth, unassisted & 83 & 60.3 & 19.2 \\
Vaginal birth, assisted & 31 & 62.0 & 17.9 \\
Unplanned caesarean section & 39 & 67.3 & 16.7 \\
Planned caesarean section, before & 24 & 67.1 & 25.5 \\
labour commenced & & & 19.5 \\
Planned caesarean section, after & 7 & 83.8 & \\
labour commenced & & & 18.8 \\
Broad birth type & & & 20.3 \\
$\quad$ Vaginal birth - any & 114 & 60.8 & \\
$\quad$ Caesarean section - any & 70 & 68.6 & \\
\hline
\end{tabular}


Table 5 Type of birth across the intervention and control groups

\begin{tabular}{lll}
\hline & Intervention (n, \%) & Control (n, \%) \\
\hline Vaginal birth, unassisted & $44(48.4 \%)$ & $39(41.9 \%)$ \\
Vaginal birth, assisted & $16(17.6 \%)$ & $15(16.1 \%)$ \\
Unplanned caesarean section & $16(17.6 \%)$ & $23(24.7 \%)$ \\
$\begin{array}{l}\text { Planned caesarean section, before } \\
\text { labour commenced }\end{array}$ & $11(12.1 \%)$ & $13(14.0 \%)$ \\
$\begin{array}{l}\text { Planned caesarean section, after } \\
\text { labour commenced }\end{array}$ & $4(4.0 \%)$ & $3(3.2 \%)$ \\
$\begin{array}{l}\text { Broad birth type } \\
\quad \text { Vaginal birth - any }\end{array}$ & \\
$\quad$ Caesarean section - any & $30(65.9 \%)$ & $54(58.1 \%)$ \\
\hline
\end{tabular}

the intervention, was less in the intervention group (AUS\$1193) than the control group (AUS\$1236), however this difference was not significant $(p=0.78)$.

Based on an odds ratio for having a caesarean section following midwife-led psycho-education, the number needed to treat (NNT) was five women treated to prevent one caesarean section (95\% CI: 3-26) (Table 7). The overall intervention cost was AUS $\$ 29$ more per woman than routine care. The incremental healthcare cost to prevent one caesarean section using this intervention was AUS\$145 (Table 7).

\section{Discussion}

Women with high childbirth fear were randomised to receive a brief psycho-education intervention from midwives who had completed a training program developed for this purpose [24]. We have previously reported that in fearful women, two antenatal psycho-education sessions with a midwife reduced high and severe score levels [25], and recommended that regardless of severity, all women fearful of birth should receive individualised support [2]. Additionally we identified that psychoeducation provided by midwives resulted in a clinically

Table 6 Health service use costs per woman. All figures in 2013 Australian dollars

\begin{tabular}{llll}
\hline & $\begin{array}{l}\text { Intervention } \\
\text { (Mean, SD) }\end{array}$ & $\begin{array}{l}\text { Control } \\
\text { (Mean, SD) }\end{array}$ & Difference \\
\hline Intervention costs & $72(24)$ & 0 & 72 \\
Health service use - total & $1193(891)$ & $1236(1264)$ & -43 \\
GP Visits & $193(112)$ & $217(101)$ & -24 \\
Obstetrician visits & $144(163)$ & $99(139)$ & 45 \\
Midwife visits & $111(92)$ & $109(90)$ & 2 \\
Nurse visits & $16(38)$ & $25(67)$ & -9 \\
Ultrasounds & $259(141)$ & $226(138)$ & 33 \\
Hospital Admissions & $238(561)$ & $216(539)$ & 22 \\
Emergency Department visits & $232(450)$ & $331(777)$ & -99 \\
Total costs & $1265(895)$ & $1236(1264)$ & 29 \\
\hline
\end{tabular}

Table 7 Cost-effectiveness estimates (95\% Cl) for the midwifeled psycho-education intervention

\begin{tabular}{ll}
\hline Difference in total cost (\$) & AUS \$29 \\
\hline $\begin{array}{l}\text { Control event risk/population expected } \\
\text { event risk for caesarean section }\end{array}$ & 0.42 \\
$\begin{array}{l}\text { Odds ratio of having a caesarean section } \\
\text { in the intervention group }(95 \% \mathrm{Cl})\end{array}$ & $0.41(0.20-0.85)$ \\
NNT $(95 \% \mathrm{Cl})$ & $5(3-26)$ \\
Cost per caesarean section prevented & AUS $\$ 145$ \\
\hline
\end{tabular}

important $8 \%$ reduction in caesarean section numbers [18]. The findings of our current analysis adds to this evidence by identifying the minimal cost incurred by the health system in offering brief psycho-education by midwives. In all, the incremental cost of preventing a caesarean section was AU\$145 using this intervention. The cost saving per avoided caesarean section could be between AUS $\$ 2449$ to $\$ 5750.48$. Refer to Table 8 for costs to the health care system per birth mode.

Our findings are similar to those of Rouhe and colleagues [20] who also conducted a randomised controlled trial testing the effect of six psycho-education group sessions with a psychologist on birth outcome in a group of Finnish primiparous women. These researchers reported improved vaginal birth rates without increasing costs compared to conventional treatment [22]. Health system differences between Finland and Australia make direct comparison difficult, as do the differences in inclusion criteria between the studies. Our results however, are reassuring and demonstrate that with minimal additional cost to health services women can receive individualised midwifery support that improves emotional health, confidence, and increases vaginal birth rates in these women.

A search of the literature revealed only one other study reporting on the cost of treating childbirth fear. Sjogren and Thomassen [13], Swedish researchers, provided individualised psychological and obstetric support to 100 women, $68 \%$ of whom originally requested caesarean section. Of these women, 57\% achieved a vaginal birth. The authors argued that the achieved reduction in caesarean section rates associated with counselling fearful pregnant women was cost neutral. Similarly our work demonstrated that following antenatal midwife psycho-education,

Table 8 Cost to the healthcare system of different types of birth, based on National Efficient Price weights and Australian Refined Diagnosis-Related Group Codes, 2013

\begin{tabular}{ll}
\hline Birth Type & Cost to healthcare system \\
\hline Unassisted vaginal birth & AUS $\$ 5000.81$ \\
Assisted vaginal birth & AUS $\$ 8301.86$ \\
Caesarean section & AUS $\$ 10,751.29$ \\
\hline
\end{tabular}


women had twice the odds of achieving a vaginal birth, and that midwife psycho-education was cost effective from a health service perspective. With this intervention, five women were required to be treated, for a total cost of AUS\$145, to prevent one caesarean section. In addition this outcome raises the prospect of longer term cost benefits potentially impacting on the number of women who would otherwise have a repeat caesarean section in a subsequent pregnancy. The four reported costing studies to date, including our study, show regardless of how costs are attributed, providing psycho-education treatment to women who are fearful of birth does not increase costs to health services and is likely to provide health services with cumulative savings over time.

\section{Implications for practice}

Consistent with earlier studies indicating fearful women are more likely to experience a caesarean section, we found fear scores at 36 weeks gestation to be predictive of the woman's birth mode, with higher scores associated to caesarean section. A recent cohort study across six European countries reported that incremental increases to antenatal fear scores are associated with the woman's subsequent birth mode [4]. Interestingly, in our study the highest scores recorded at 36 weeks of pregnancy were by women who spontaneously laboured prior to their planned caesarean section. This is an important finding alerting maternity providers to the level of preparation and support women may require to reassure them of a safe outcome, and explaining that planning a caesarean section alone is not a solution to their fear. Swedish experts [30] have previously advised that prescription or agreement for a caesarean section does not alleviate women's fear. Our findings highlight the need for clear birth planning and an exploration of women's understanding of alternate pathways that might occur leading up to the birth. This preparation allows women to anticipate and plan for possible eventualities. One aspect of the BELIEF psycho-education framework [24] was for midwives to encourage women to envisage how they wanted their birth to be and also what it might look like if other factors impacted and changed their desired course. Taking this approach assisted women to think through strategies they felt they could manage whilst also providing them with a 'Plan B', and in some cases a 'Plan C' if needed.

The positive effects of this midwife intervention on women's reduced fear levels, higher vaginal birth rates and minimal cost to health organisations to reduce caesarean section rates makes investment in midwife psycho-education attractive to health services. Additionally, combining psycho-education for women with childbirth fear and cost effective midwifery caseload care is likely to produce even higher benefits for maternity service organisations and for women with childbirth fear [31-34]. To assist screening of women with childbirth fear the validated two item Fear of Birth scale (FOBs) could also be used, as it is time-efficient for clinicians, and aligns to the widely used and accepted W-DEQ A tool [35].

\section{Limitations and strengths}

Costs were attributed to health service use and therefore women's personal out of pocket expenditure were not known. Records of service use was reliant on women's self-report; however women carry a personal pregnancy health record and were not reliant on recall alone. We cannot be certain of the generalisability of the findings as approximately $30 \%$ of Australian women receive private obstetric care. We recruited from public hospitals only. Also only women who spoke English were included leaving non-English speaking women marginalised and; women of lower education and income were more likely to drop out of the study [25]. Lastly, despite a number of engagement strategies only $54 \%$ of women returned data at 6 weeks following birth, although this rate is comparable to similar studies involving fearful women [22].

Although the costs reported from this study indicate the potential for significant cost savings as a result of reduced caesarean sections, a previous study reporting the total costs of this intervention, including the costs of birth mode, did not report a significant difference in total costs between the two arms [23]. This nonsignificant result may be due to a low sample size yielding insufficient power to detect a difference in cost between the two groups, with the study being powered to detect a difference in fear of birth scores.

The strength of the work, however, is that participants were enrolled from three hospitals allowing for sample diversity. We also did not place exclusions on women's participation in respect of obstetric risk (for example women of any parity, singleton and multiple pregnancies, any type of previous birth). In addition Australian women do not have routine access to treatment for childbirth fear that is available in some other countries. We can be confident, therefore, that the differences between groups were a result of inclusion in the study and were unlikely to be attributable to an alternate childbirth fear intervention. Furthermore, despite women being randomised by a computerised system, women in the psycho-education group (intervention) had higher WDEQ A scores than controls at baseline making the study result more encouraging.

\section{Conclusion}

Following antenatal midwife psycho-education for women with high levels of childbirth fear, vaginal birth 
rates improved and caesarean section rates decreased compared with women who received standard antenatal care and a decision-aid booklet. For every five women who received midwife counselling, one caesarean section was averted. The incremental healthcare cost to prevent one caesarean section using this intervention was only AUS\$145. Based on the result of this study it is recommended that maternity services invest in and support midwives to deliver psycho-education to women with childbirth fear. This will afford fearful women an opportunity to develop positive feelings of anticipation for their birth and subsequently improve their chance of achieving vaginal birth.

\begin{abstract}
Abbreviations
AUS: Australia; BELIEF: Birth Emotions and Looking to Improve Expectant Fear; CS: Caesarean section; FOBs: Fear of Birth scale; ICER: Incremental costeffectiveness ratio; NNT: Number needed to treat; PEER: Population expected event risk; W-DEQ A: Wijma Delivery Expectancy/Experience Questionnaire Antenatal
\end{abstract}

\section{Acknowledgements}

Not applicable.

\section{Funding}

The BELIEF study was funded by the National Health and Medical Research Council (NHMRC Grant ID APP1025099). The NHMRC played no role in the design of the study, or the collection, analysis, and interpretation of data or in writing the manuscript.

\section{Availability of data and materials}

The datasets supporting the conclusions of this article are included within the article.

\section{Authors' contributions}

JT, EC, JF, JG \& DKC conceptualized the manuscript. JT drafted the manuscript. EC conducted statistical analysis. JT, EC, JF, JG \& DKC each contributed to analysis of data and critically reviewed drafts of the manuscript. $\int T$ coordinated revision of the manuscript. JF is the CIA of the BELIEF project, JG \& DKC are Cls on the team and JT was the project manager. All authors are equally accountable for all aspects of the work and have read and approved the final manuscript.

\section{Ethics approval and consent to participate}

Approval to conduct the study was granted from Griffith University Human Research Ethics Committee (NRS/45/11/HREC) and Queensland Health (HREC/11/QGC/162). After ethical approval was granted, women in their second trimester of pregnancy were approached in antenatal clinics to participate, were provided verbal and written advice of the study, with written consent received from women who agreed to participate. The full study protocol is available [24].

\section{Consent for publication}

Not applicable.

\section{Competing interests}

The authors declare they have no competing interests.

\section{Publisher's Note}

Springer Nature remains neutral with regard to jurisdictional claims in published maps and institutional affiliations.

\section{Author details}

1 School of Nursing and Midwifery \& Menzies Health Institute Queensland, Griffith University, University Drive, Meadowbrook 4131, Australia. ${ }^{2}$ Australian Institute of Tropical Health and Medicine, James Cook University, Townsville 4812, Australia. ${ }^{3}$ Gold Coast University Hospital, Southport 4215, Australia.
${ }^{4}$ Office of the Chief Nursing and Midwifery Officer, Queensland Department of Health, Brisbane 4001, Australia.

Received: 22 July 2016 Accepted: 3 July 2017

Published online: 11 July 2017

\section{References}

1. O'Connell, M., et al., Worldwide prevalence of tocophobia in pregnant women: systematic review and meta-analysis. Acta Obstet Gynecol Scand, 2017.

2. Toohill, J., et al., A cross sectional study to determine utility of childbirth fear screening in maternity practice - an Australian perspective. Women and Birth, 2015.

3. Fenwick J. et al. Sources, responses and moderators of childbirth fear in Australian women: a qualitative investigation Midwifery. 2015;31(1):239-46.

4. Ryding $E$, et al. Fear of childbirth and risk of cesarean delivery: a cohort study in six European countries. Birth. 2015;42(1):48-55.

5. Rouhe $\mathrm{H}$, et al. Mental health problems common in women with fear of childbirth. BJOG. 2011;118:1104-11.

6. Jokic-Begic N, Zigic L, Rados S. Anxiety and anxiety sensitivity as predictors of fear of different patterns for nulliparous and parous women. J Psychosom Obstet Gynecol. 2014;35(1):22-8.

7. Raisanen S, et al. Fear of Childbirth Predicts Postpartum Depression: A Population-Based Analysis of 511422 Singleton Births in Finland. BMJ Open. 2013;3(11):e004047. doi:10.1136/bmjopen-2013-004047.

8. Fisher C, Hauck Y, Fenwick J. How social context impacts on women's fears of childbirth: a western Australian example. Soc Sci Med. 2006;63(1):64-75.

9. Ryding E. Investigation of 33 women who demanded a caesarean section for personal reasons. Acta Obstet Gyn Scan. 1993;72(4):280-5.

10. Toohill, J., et al., Prevalence of childbirth fear in an Australian sample of pregnant women. BMC Pregnancy and Childbirth 2014. 14:275.

11. Fenwick J, et al. Pre- and postpartum levels of childbirth fear and the relationship to birth outcomes in a cohort of Australian women. J Clin Nurs. 2009;18:667-77.

12. Haines $\mathrm{H}$, et al. Cross-culturalcomparisonoflevelsofchildbirthrelatedfearinanAustralian and Swedishsample. Midwifery. 2011;27:560-7.

13. Sjogren B, Thomassen P. Obstetric outcome in 100 women with severe anxiety over childbirth. Acta Obstet Gynecol Scand. 1997;76:948-52.

14. Sydsjo G, et al. Obstetric outcome for women who received individualized treatment for fear of childbirth during pregnancy. Acta Obstet Gynecol Scand. 2012:91:44-9.

15. Koechlin, F., L. Lorenzoni, and P. Schreyer, Comparing Price Levels of Hospital Services Across Countries: Results of Pilot Study, OECD Health Working Papers, No. 53, OECD Publishing. Retrieved 22nd January, 2014, from http://dx.doi.org/10.1787/5km91p4f3rzw-en. 2010.

16. Gibbons, L., et al., The Global Numbers and Costs of Additionally Needed and Unnecessary Caesarean Sections Performed per Year: Overuse as a Barrier to Universal Coverage. World Health Report. Background Paper, No 30. World Health Organisation. Retrieved 22nd January, 2014, from http:// www.who.int/healthsystems/topics/financing/healthreport/30C-sectioncosts. pdf. 2010

17. OECD., "Caesarean sections", in OECD., Health at a Glance 2015: OECD Indicators, OECD Publishing, Paris. DOl: http://dx.doi.org/10.1787/health_ glance-2015-37-en. 2015.

18. Fenwick, J., et al., Effects of a midwife psycho-education intervention to reduce childbirth fear on women's birth outcomes and postpartum psychological wellbeing BMC Pregnancy and Childbirth, 2015. 15:284).

19. Halvorsen L, Nerum H, Sorlie T. Does counsellor's attitude influence change in a request for a caesarean in women with fear of birth? Midwifery. 2010; 26:45-52.

20. Rouhe $\mathrm{H}$, et al. Obstetric outcome after intervention for severe fear of childbirth in nulliparous women - randomised trial. BJOG. 2013;120:75-84.

21. Saisto $T$, et al. A randomized controlled trial of intervention in fear of childbirth. Obstetrics and Gynecology. 2001;98(5):820-6.

22. Rouhe $\mathrm{H}$, et al. Life satisfaction, general well-being and costs of treatment for severe fear of childbirth in nulliparous women by psychoeducative group or conventional care attendance. Acta Obstet Gynecol Scand. 2015; 94:527-33.

23. Turkstra $\mathrm{E}$, et al. An economic evaluation alongside a randomised controlled trial on psycho-education counselling intervention offered by midwives to 
address women's fear of childbirth in Australia. Sexual \& Reproductive Healthcare. 2017;11:1-6.

24. Fenwick, J., et al., Study protocol for reducing childbirth fear: a midwife-led psycho-education intervention. BMC Pregnancy and Childbirth, 2013. 13190.

25. Toohill J, et al. A randomized controlled trial of a psycho-education intervention by midwives in reducing childbirth fear in pregnant women. Birth. 2014;41(4):384-94.

26. Wijma K, Wijma B, Zar M. Psychometric aspects of the W-DEQ; a new questionnaire for the measurement of fear of childbirth. J Psychosom Obstet Gynecol. 1998;19(2):84-97.

27. Authority IHP. National Hospital Cost Data Collection, public hospitals cost report, round 19 (financial year 2014-15). Sydney: IHPA; 2017.

28. CEBM, Number needed to treat (NNT). [cited 2016 20th May]; Available from: http://www.cebm.net/number-needed-to-treat-nnt/. 2016.

29. Sackett D, Deeks J, Altman D. Down with odds ratios! Evidenced Based Medicine. 1996; (6):164-6.

30. Hildingsson I, et al. A longitudinal survey of childbirth-related fear and associated factors. JOGNN. 2011;40(5):532-43.

31. Toohill J, et al. A non-randomised trial investigating the cost-effectiveness of midwifery group practice compared with standard maternity care arrangements in one Australian hospital. Midwifery. 2012;28(6):e874-9.

32. Bernitz $\mathrm{S}$, Aas $\mathrm{E}$, Oian $\mathrm{P}$. Economic evaluation of birth care in low-risk women. A comparison between a midwife-led birth unit and a standard obstetric unit within the same hospital in Norway.A randomised controlled trial. Midwifery. 2012;28:591-9.

33. Sandall J, et al. Midwife-led continuity models versus other models of care for childbearing women Cochrane Library. 2016; doi:10.1002/14651858. CD004667.pub5.

34. Tracy $\mathrm{S}$, et al. Caseload midwifery care versus standard maternity care for women of any risk: M@NGO, a randomised controlled trial. Lancet. 2013; 382(9906):1723-32

35. Haines, $\mathrm{H}_{\text {., }}$ et al., Identifying women who are afraid of giving birth: A Comparison of the Fear of Birth Scale with the WDEQ-A in a large Australian cohort Sexual and Reproductive Health Care.n, 2015. 6(4): p. 204-210.

\section{Submit your next manuscript to BioMed Central and we will help you at every step:}

- We accept pre-submission inquiries

- Our selector tool helps you to find the most relevant journal

- We provide round the clock customer support

- Convenient online submission

- Thorough peer review

- Inclusion in PubMed and all major indexing services

- Maximum visibility for your research

Submit your manuscript at www.biomedcentral.com/submit

) Biomed Central 